\title{
ABSTRACT
}

\section{Evaluation of Pharmaceutical Intervention at Hepatitis C Medication Therapy Adherence Clinic in North Borneo, Malaysia (Epic MTAC)}

Bee Keng Law”, Euginie Tracy Wong, Qiao Wei Liew, Zhi Sam Heng

Department of Pharmacy,

Hospital Queen Elizabeth, Kota Kinabalu,

Sabah, Malaysia

\section{*Corresponding author's email:}

lawbkg@gmail.com
Introduction: Hepatitis C virus (HCV) is a worrying public health issue worldwide. The introduction of direct-acting antiviral agents (DAAs) brings revolution to HCV treatment. Pharmacists' role in Malaysia is significant since the implementation of Medication Therapy Adherence Clinic (MTAC). This study aims to determine the sustained virological response (SVR12) for HCV patients treated with Sofosbuvir and Daclatasvir and/or Ribavirin. Besides, it evaluates adherence rate, types of pharmaceutical intervention and physicians' acceptance rate. Methods: A retrospective cohort study on hepatitis $C$ patients who received DAAs-based treatment in 2018 at North Borneo government facilities. Results: Hepatitis C MTAC recruited 53 out of 56 patients. The overall SVR1 2 was $91 \%$. SVR12 for MTAC patients was better than non-MTAC group but the differences were statistically not significant $(92.45 \%$ vs $66.67 \% ; p>0.05)$. The overall adherence rate towards treatment was 98\%. Although MTAC patients showed higher adherence rate, there was no significant difference among the two groups (99\% vs $89 \%$; $p=0.639$ ). During 279 MTAC visits, a total of 43 drug-drug interactions (DDIs) were identified. The majority $(98 \%)$ rated as potential DDIs that were intervened by either close monitoring (89\%), dosage adjustment (7\%), medication replacement $(2 \%)$ or medication cessation (2\%) with $100 \%$ acceptance rate. Among the 164 medications reviewed, Amlodipine and Efavirenz were the most commonly involved drugs. Adverse drug reactions (ADRs) were 
Borneo Journal of Medical Sciences (BJMS), Special Issue, Volume 3, March 2020: 9 - 10

detected in approximately one-third (30\%) of the MTAC patients. Three most frequently reported ADRs were fatigue (14\%), dizziness (14\%) and headache (7\%). Conclusion: The contrariety of sample size between MTAC and the non-MTAC group is the limitation of this study, therefore unable to accurately reflect the significance of MTAC. However, the role of MTAC pharmacists cannot be denied because they are not only serving as a dispenser but actively involved in disease management by ensuring adherence and advocate pharmacovigilance. 